\title{
El terremoto de Lorca de 11 de mayo de 2011 y la sismicidad de la región
}

\author{
JosÉ MANUEL MARTÍNEZ SOLARES ${ }^{1,2}$, JUAN VICENTE CANTAVELLA NADAL ${ }^{1}$, \\ LUIS CABAÑAS RODRÍGUEZ ${ }^{1} \&$ JOSÉ FRANCISCO VALERO ZORNOSA ${ }^{1}$ \\ ${ }^{1}$ Instituto Geográfico Nacional, Ministerio de Fomento \\ ${ }^{2}$ Dpto. de Geofísica y Meteorología, Universidad Complutense de Madrid \\ jmmsolares@fomento.es
}

Recibido: $22 / 06 / 2012$

Aceptado: 11/10/2012

\section{Resumen}

Se describe la serie sísmica de Lorca de 2011, presentando sus parámetros de localización y determinando de forma precisa el tensor momento sísmico de los sismos principales. Para toda la secuencia sísmica se analizan sus características mediante el estudio de su distribución temporal y espacial, comparándola con anteriores terremotos. Se resumen los datos macrosísmicos recogidos en la zona así como los valores de la PGA y se realiza una revisión de la sismicidad regional, tanto de los terremotos históricos como de los instrumentales, llegando a modificar algunos de sus parámetros.

Palabras clave: Terremoto de Lorca, series sísmicas, intensidad macrosísmica, sismicidad.

\section{The 11 May 2011 Lorca earthquake and the seismicity of the region}

\begin{abstract}
Lorca 2011 seismic series is described presenting its location parameters and the seismic moment tensor of its main events. We analyse its features by means of the whole seismic sequence temporal and spatial distribution, and we compare it with previous seismic series in the same region. Macroseismic data and PGA values gathered in this area are summarized in this paper. In addition, after a thorough revision of the regional seismicity for both historic and instrumental events, we make some modifications in the seismic catalogue.
\end{abstract}

Keywords: Lorca earthquake, seismic series, macroseismic intensity, seismicity.

Sumario: Introducción. 1. Terremotos del 11 de mayo de 2011. 1.1 Localización. 1.2 Tensor momento sísmico. 1.3 Registros de aceleración. 1.4 Información macrosísmica. 1.5 Características de la serie sísmica. 2. Sismicidad de la región. 2.1. Periodo histórico. 2.2. Periodo instrumental. 3. Conclusiones Agradecimientos. Referencias bibliográficas.

\section{Referencia normalizada}

Martínez Solares, J.M., Cantavella Nadal, J.V., Cabañas Rodriguez, L., Valero Zornosa, J.F. (2012). El terremoto de Lorca de 11 de mayo de 2011 y la sismicidad de la región. Física de la Tierra, Vol. $24,17-40$. 


\section{Introducción}

Desde el terremoto de 19 de abril de 1956 (5,0 mbLg) ocurrido en Albolote (Granada) con once muertos y el más reciente de 1969 (7,8 Mw) al SW del Cabo San Vicente con cuatro, no había sucedido en España ninguno que ocasionase víctimas mortales como el acaecido en Lorca (Murcia) el 11 de mayo de 2011 que produjo nueve muertos en la ciudad, todos ellos debido al desprendimiento de elementos de fachada de los edificios de la propia ciudad de Lorca.

Podemos considerar que el nivel de sismicidad de la región de Murcia es moderado, sobresaliendo el terremoto del periodo histórico sucedido en Lorca en 1674 que tuvo una intensidad de VIII, con un número de víctimas próximo a la treintena. No obstante, este terremoto de 2011 se suma a un incremento observado de la actividad sísmica de la región en los últimos 12 años en los que se han registrado terremotos de magnitudes 4,7 mbLg (Mula, 1999), 5,0 Mw (Bullas, 2002) y 4,8 Mw (Aledo, 2005). El terremoto de Lorca de 2011 (5,1 Mw) supera en magnitud a los anteriores e iguala el grado VII en intensidad máxima sentida con el de Aledo de 2005, sin embargo la población afectada en Lorca fue muy superior, así como la cantidad de edificios afectados, pues el casco urbano de Lorca concentra unos 60.000 habitantes, mientras que Zarcilla de Ramos, donde se alcanzó el grado máximo de VII supera escasamente los 1.000 habitantes.

Este artículo presenta los resultados obtenidos en los parámetros de localización de la serie y se calcula de nuevo el tensor momento sísmico para los tres mayores terremotos utilizando el máximo de estaciones disponibles. También se realiza un análisis comparativo, espacial y temporal, de las características de la serie sísmica respecto a sismos anteriores. Por otro lado, se han evaluado los datos macrosísmicos recogidos en la zona junto con los valores de aceleración registrados, poniendo de manifiesto su importancia a la hora de disponer de información rápida y fiable en la gestión de la catástrofe. Asimismo se realiza un análisis del conjunto de la sismicidad regional, exponiendo brevemente los aspectos más significativos de los mayores terremotos, tanto de la época histórica como de la instrumental y aportando una nueva evaluación de los parámetros de algunos de ellos.

\section{Terremotos del 11 de mayo de 2011}

La serie sísmica de 2011, aparte de sus numerosas réplicas, la podemos caracterizar con los tres terremotos de mayor magnitud, un sismo premonitorio de 4,5 $\mathrm{Mw}$ sentido en Lorca con intensidad VI (EMS98), generando bastante alarma en la población, que poco después se vio sorprendida por un terremoto de mayor magnitud, 5,1 Mw, con intensidad VII y una réplica, la de mayor magnitud, 3,9 Mw, que sucedió unas cuatro horas después del principal. 


\subsection{Localización}

La localización hipocentral de toda la serie se ha calculado con las estaciones sísmicas tanto del IGN como de otras instituciones (IAG, ROA-UCM, UA) ${ }^{1}$, limitando su uso a las situadas a distancias inferiores a los $4^{\circ}$. Asimismo, dada la proximidad de los equipos de aceleración, sus tiempos de llegada también han sido utilizados en el cálculo de los terremotos principales, sobresaliendo el acelerógrafo de Lorca que situado muy próximo al epicentro, ha permitido un cálculo del hipocentro con un margen de error muy pequeño. El modelo de corteza utilizado ha sido el general del IGN (Mezcua \& Martínez Solares, 1983). Los resultados del cálculo de los parámetros de localización y tamaño de los tres terremotos mayores de la serie, el premonitorio, el principal y la mayor réplica son los que figuran en la tabla 1 y figura 1.

Tabla 1. Localización y tamaño de los terremotos premonitorio, principal y mayor réplica.

\begin{tabular}{|c|c|c|c|c|c|}
\hline Fecha & $\begin{array}{c}\text { Hora } \\
(\mathrm{GMT})\end{array}$ & Latitud & Longitud & $\begin{array}{c}\text { Prof } \\
(\mathrm{km})\end{array}$ & $\mathrm{Mw}$ \\
\hline 11-mayo-2011 & $15: 05: 13.5$ & 37,7196 & $-1,7076$ & 2 & 4,5 \\
\hline 11-mayo-2011 & $16: 47: 26.0$ & 37,7175 & $-1,7114$ & 4 & 5,1 \\
\hline 11-mayo-2011 & $20: 37: 45.4$ & 37,7308 & $-1,7012$ & 4 & 3,9 \\
\hline
\end{tabular}

Los terremotos están localizados muy próximos entre sí, aproximadamente entre 4,5 y 5,0 km al norte de la ciudad de Lorca. También las profundidades de los focos sísmicos presentan poca diferencia, si bien este parámetro, al estar más condicionado a causa del modelo de estructura, presenta una mayor incertidumbre.

\subsection{Tensor momento sísmico}

El IGN determinó de forma automática el tensor momento sísmico de los tres mayores eventos de la serie siguiendo el método detallado en Rueda \& Mezcua (2005). De este modo se pudo conocer la magnitud momento y el mecanismo focal de estos tres terremotos a los pocos minutos de su ocurrencia.

En este trabajo se calcula de nuevo el tensor momento sísmico de los tres haciendo uso de todos los datos disponibles provenientes de las instituciones anteriormente señaladas. El cálculo se ha realizado mediante el ajuste en el dominio del tiempo de la forma de onda completa a sismogramas sintéticos. Para ello se ha utilizado el paquete de software desarrollado por Herrmann \& Ammon (2002).

${ }^{1}$ IGN: Instituto Geográfico Nacional. IAG: Instituto Andaluz de Geofísica. ROA: Real Observatorio de la Armada. UCM: Universidad Complutense de Madrid. UA: Universidad de Alicante. 


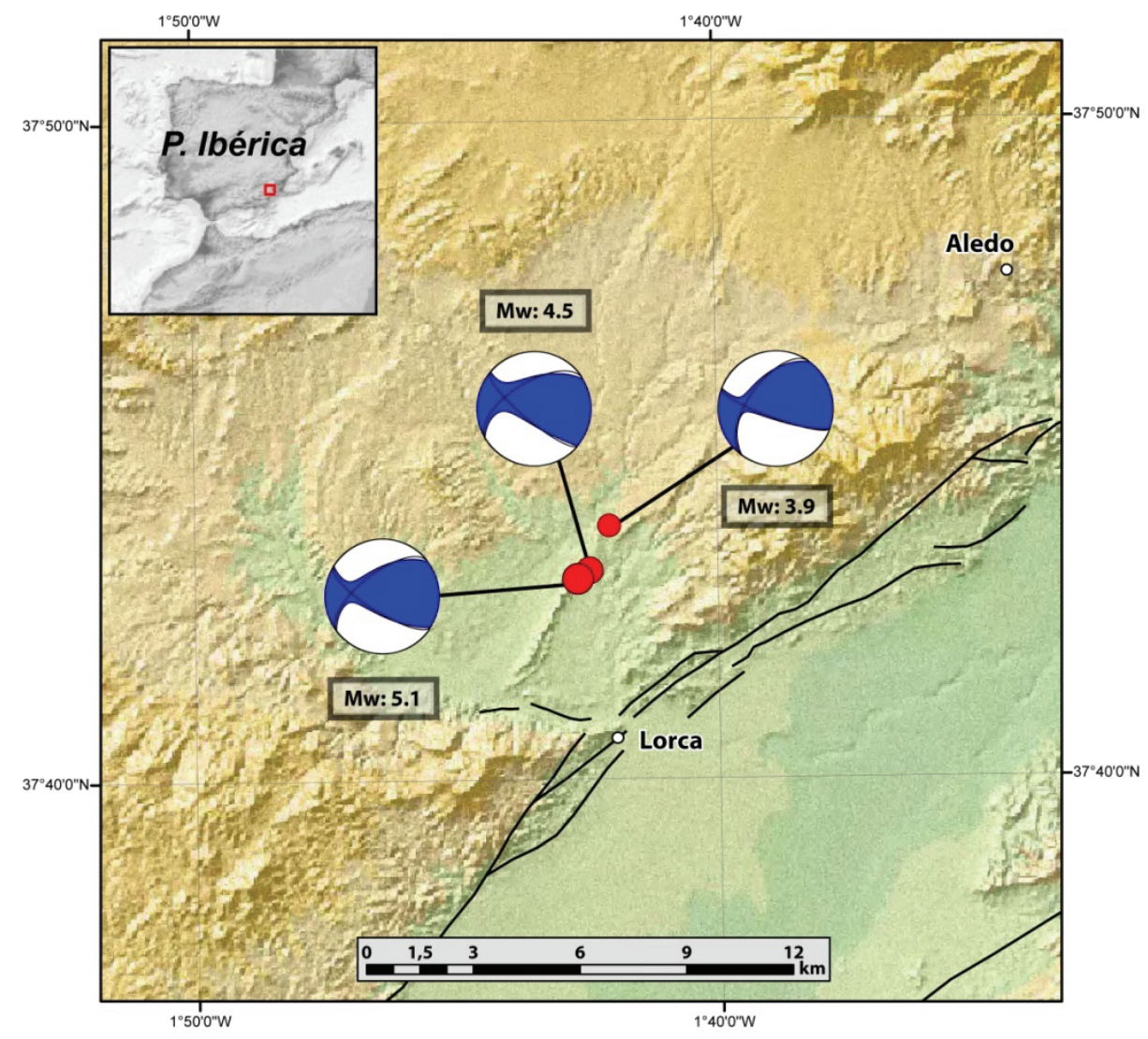

Fig. 1. Localización de los tres terremotos principales, mecanismo focal y magnitud Mw.

El modelo de corteza usado para generar las funciones de Green es el propuesto en Rueda \& Mezcua (2005) para la Península Ibérica. La profundidad del centroide se estimó realizando la inversión para todo el rango de profundidades en intervalos de $1 \mathrm{~km}$, tomando como mejor solución aquella para la cual se obtenía un mejor ajuste y una mayor componente de doble par. En la inversión se supone que la fuente no tiene componente isótropa.

En la Tabla 2 se recogen los principales parámetros de la inversión del tensor momento sísmico para estos tres eventos. En la Figura 2 se presenta para el terremoto principal: a) el ajuste entre los sismogramas observados y sintéticos para la profundidad elegida, b) la calidad del ajuste de los datos observados con los sismogramas sintéticos en función de la profundidad del centroide y c) un mapa con las estaciones utilizadas para la inversión del tensor momento sísmico. 


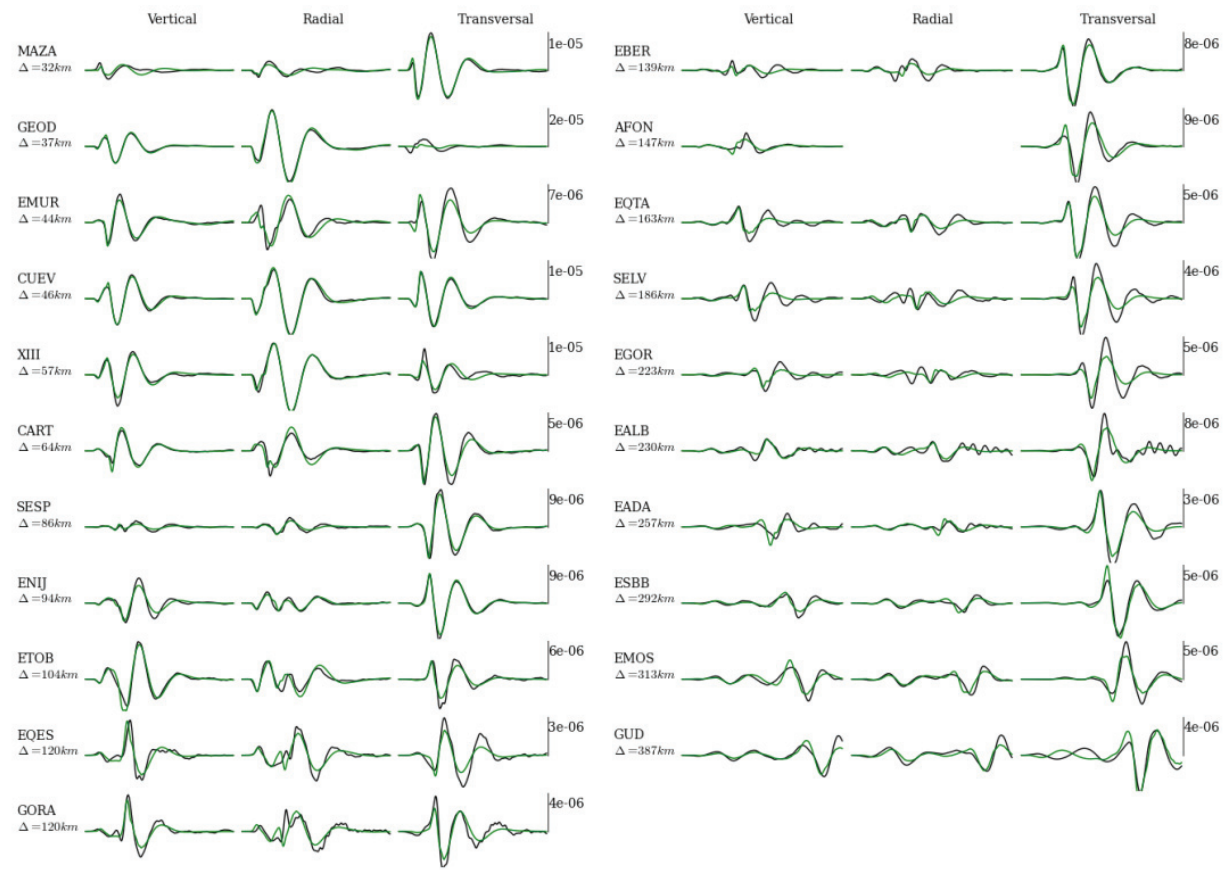

(a)

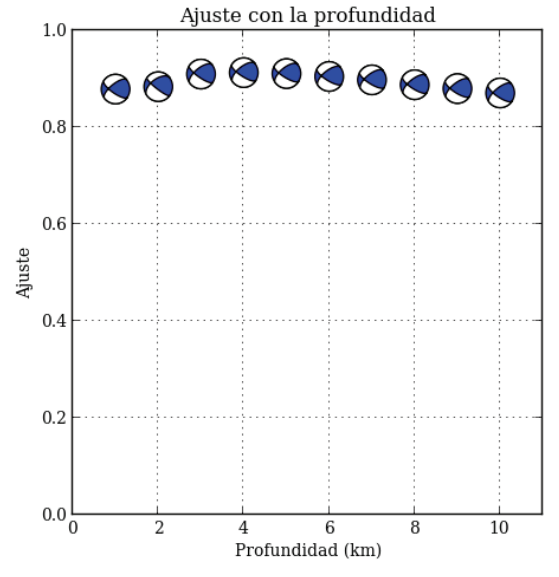

(b)

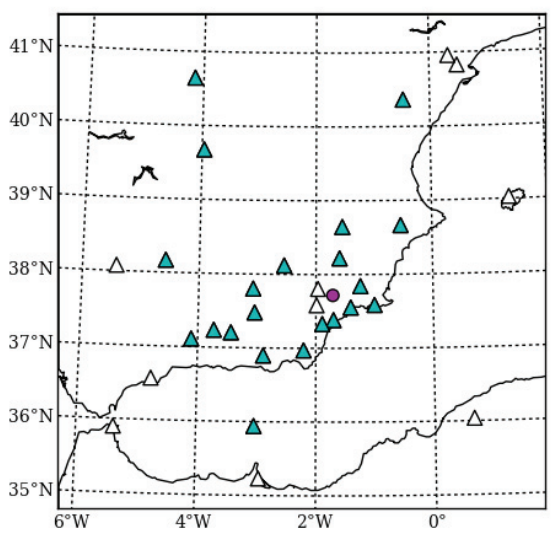

(c)

Fig. 2. Para el terremoto principal 5,1 Mw: a) ajuste entre sismogramas observados y sintéticos. b) calidad del ajuste en función de la profundidad del centroide. c) estaciones de banda ancha usadas en el cálculo (triángulos oscuros). 
Como se puede deducir de la figura $1 \mathrm{y}$ tabla 2, el mecanismo de estos tres eventos corresponde a un movimiento de falla inversa con ligera componente de desgarre, coincidiendo con las características de esta parte de la falla de Alhama de Murcia, cuya longitud se puede estimar en $85 \mathrm{~km}$, si bien está segmentada en diversos tramos con direcciones algo diferentes entre sí (Martínez Diaz, 2002). Asimismo, las profundidades atribuidas al centroide de estos tres sismos son compatibles con las profundidades hipocentrales expuestas anteriormente.

Tabla 2. Parámetros principales del cálculo del tensor momento sísmico de los tres mayores terremotos de la serie.

\begin{tabular}{|l|l|l|c|c|c|l|l|}
\hline $\begin{array}{l}\text { Fecha } \\
\text { Hora }\end{array}$ & $\begin{array}{l}\text { Plano nodal 1 } \\
\text { (strike, dip, } \\
\text { slip) }\end{array}$ & $\begin{array}{l}\text { Plano nodal 2 } \\
\text { (strike, dip, } \\
\text { slip) }\end{array}$ & $\begin{array}{c}\text { Mo } \\
(\mathrm{din} \cdot \mathrm{cm})\end{array}$ & Mw & $\begin{array}{l}\text { Prof. } \\
\text { centroide } \\
(\mathrm{km})\end{array}$ & $\begin{array}{l}\% \\
\text { Doble } \\
\text { Par }\end{array}$ & Ajuste \\
\hline $\begin{array}{l}2011 / 05 / 11 \\
15: 05: 13\end{array}$ & $(129,71,135)$ & $(238,48,26)$ & $6,04 \mathrm{E}+22$ & 4,5 & 3 & $76 \%$ & $88 \%$ \\
\hline $\begin{array}{l}2011 / 05 / 11 \\
16: 47: 26\end{array}$ & $(122,66,138)$ & $(232,53,31)$ & $5,82 \mathrm{E}+23$ & 5,1 & 4 & $89 \%$ & $92 \%$ \\
\hline $\begin{array}{l}2011 / 05 / 11 \\
20: 37: 45\end{array}$ & $(110,75,134)$ & $(215,46,21)$ & $7,96 \mathrm{E}+21$ & 3,9 & 3 & $93 \%$ & $77 \%$ \\
\hline
\end{tabular}

\subsection{Registros de aceleración}

El terremoto principal de la serie fue registrado en un total de 17 instrumentos de la red acelerográfica del IGN, la mayoría situados en la zona epicentral, pero también algunos de ellos a distancias considerables como Albolote o Jaén (más de $180 \mathrm{~km}$ ), con valores de aceleración pico justo en el límite de la perceptibilidad humana, del orden de $2-3 \mathrm{~cm} / \mathrm{s}^{2}$, que bien podrían corresponder con un valor de intensidad macrosísmica de II en la escala EMS98.

En la figura 3 pueden verse las estaciones de aceleración del IGN, en la región de Murcia y provincias contiguas, que registraron el terremoto principal. En cada una se ha anotado el valor de la PGA máxima (horizontal) obtenida.

El acelerógrafo del IGN situado en Lorca, próximo a su Ayuntamiento, registró una aceleración pico horizontal de $0,36 \mathrm{~g}$ durante el terremoto principal y de $0,27 \mathrm{~g}$ en el terremoto premonitorio, ambas en la componente $\mathrm{N} 30 \mathrm{~W}$, aproximadamente perpendicular a la dirección de la ruptura. El terreno sobre el que se sitúa esta estación puede ser catalogado como un suelo duro (tipo II según NCSE-02, tipo B según EC-8), por lo que la aceleración bien pudo alcanzar picos mayores en otras zonas de la ciudad con terrenos algo más blandos. A pesar de las magnitudes moderadas de estos dos terremotos, factores como la proximidad del epicentro $(5-6 \mathrm{~km})$, la superficialidad de la fuente sísmica $(2-4 \mathrm{~km})$ y también efectos de campo cercano como la polarización del movimiento y seguramente la directividad (Rueda et al. 2011), podrían explicar esta alta aceleración. No obstante, sólo 
un edificio con estructura de hormigón colapsó y según los informes preliminares pudo ser debido a su mal diseño estructural.

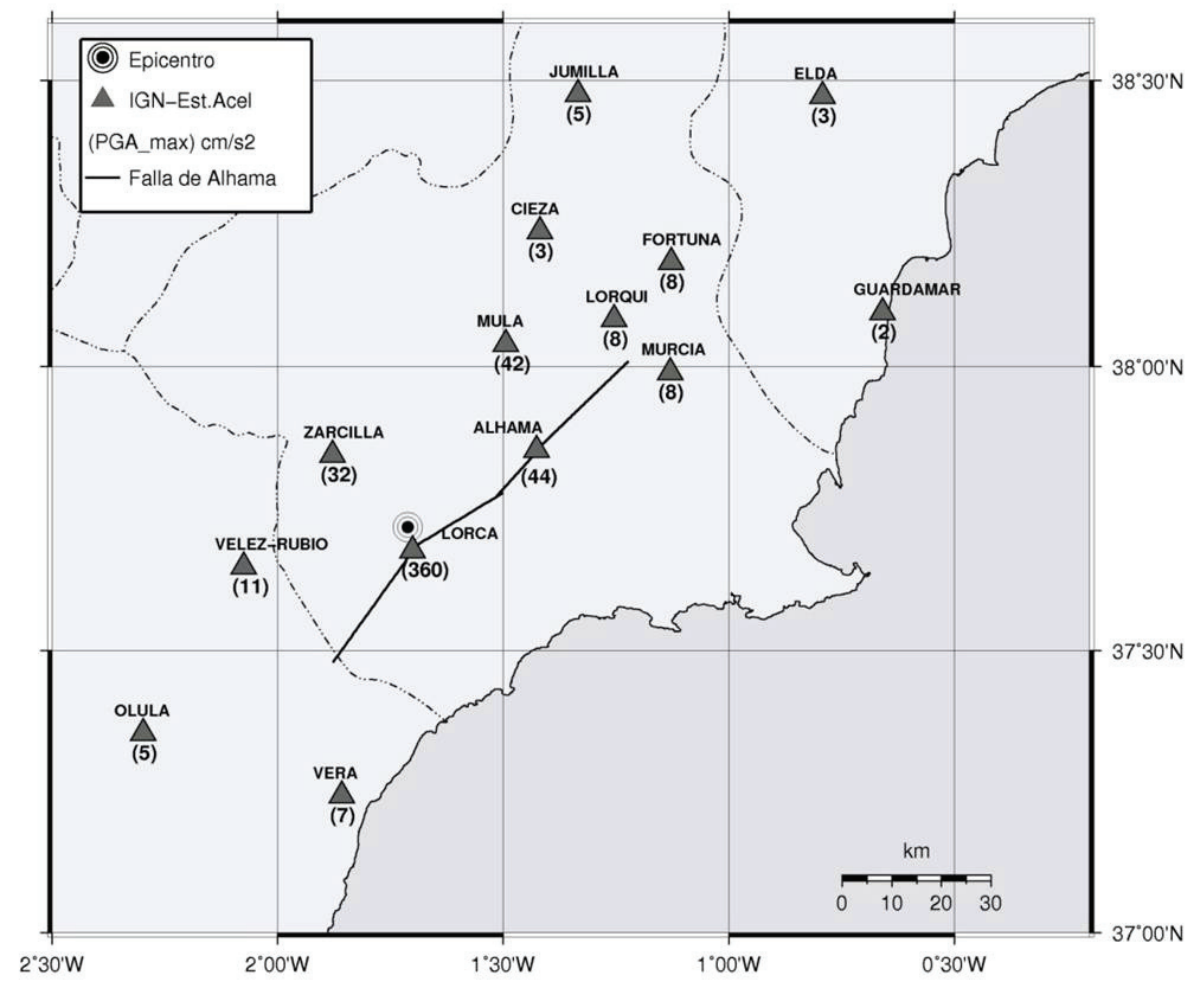

Fig. 3. Distribución geográfica de las estaciones de la red de aceleración del IGN que registraron el terremoto principal en la zona más próxima. Entre paréntesis se muestran los valores de PGA horizontal máxima $\left(\mathrm{en} \mathrm{cm} / \mathrm{s}^{2}\right.$ ) de cada estación.

En la figura 4 se muestran, para algunas de las estaciones representadas en la figura anterior, las componentes horizontales de aceleración en las que se obtuvo la máxima PGA y que fueron registradas durante el terremoto principal. Éstas se han dibujado en distinta escala vertical, (aceleración en $\mathrm{cm} / \mathrm{s}^{2}$ ), dada la diferencia de amplitud entre los registros y en la misma escala horizontal (tiempo no absoluto en s), aunque en distintos intervalos de tiempo, con el fin de poder destacar la parte más prominente de cada registro y las frecuencias que contienen. En la página web del IGN (www.ign.es) se pueden obtener los valores máximos registrados para las tres componentes de 13 terremotos de la serie. 


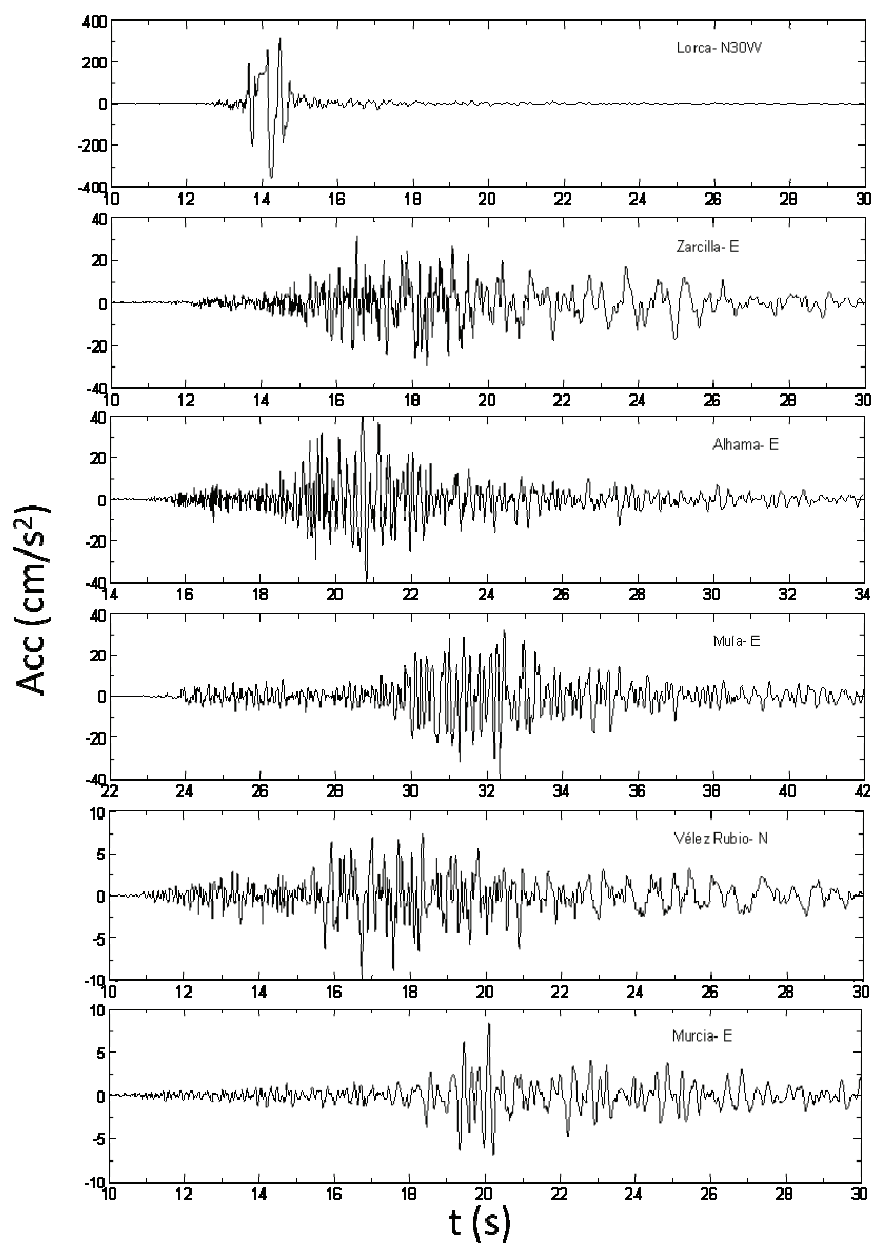

Fig. 4. Componentes de aceleración horizontal de máxima PGA, registrados en las estaciones más cercanas al epicentro, correspondientes al terremoto principal.

Actualmente, la normativa sismorresistente española NCSE-02 asigna a Lorca una aceleración sísmica básica $\mathrm{a}_{\mathrm{b}}=0,12 \mathrm{~g}$ (figura 5). Esta aceleración no puede ser comparada directamente con los valores de aceleración registrados en la ciudad (PGA horizontal - media geométrica $=0,24 \mathrm{~g}$ ), ya que por una parte esta aceleración básica fue calculada como una aceleración 'característica', no como la máxima, y por otra parte porque estaría asociada a una probabilidad de excedencia concreta $(10 \%$ en 50 años). En la figura 5 se presentan los valores actuales de $\mathrm{a}_{\mathrm{b}}$ para toda la región de Murcia, donde se puede observar que las mayores aceleraciones, 0,15-0,16 g, no corresponden a Lorca, sino a Murcia capital y a las localidades situadas a su NE. En cualquier caso, una nueva revisión del mapa de 
peligrosidad de España, próxima a su publicación, modificará los valores del mapa.

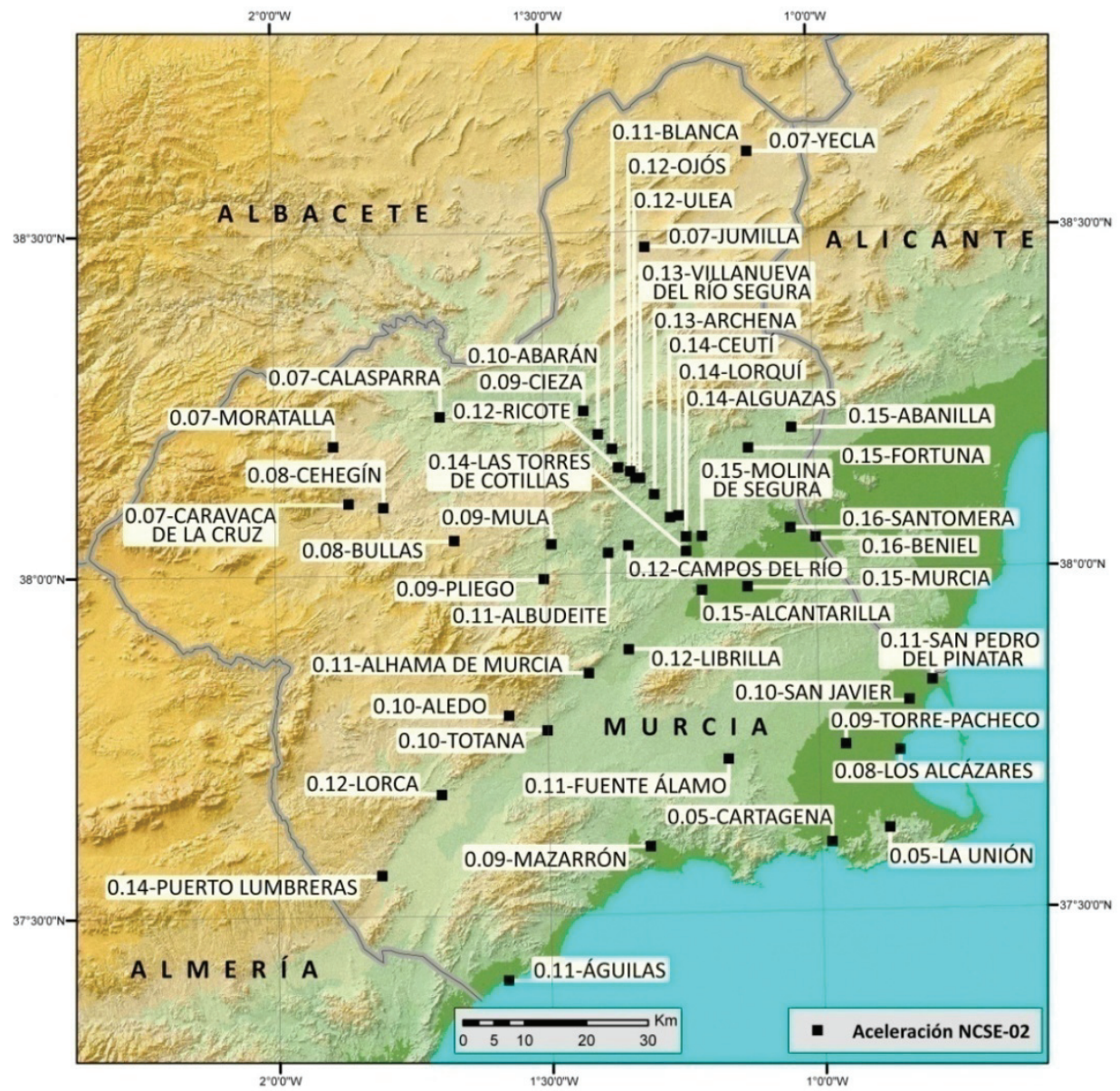

Fig. 5. Valores de la aceleración sísmica básica $a_{b}$ para la región de Murcia según la NCSE-02.

\subsection{Información macrosísmica}

El terremoto principal del día 11 de mayo de 2011, que estuvo localizado a unos cinco kilómetros de la ciudad de Lorca, registró en esta ciudad una intensidad máxima de VII (escala EMS98). El sismo fue sentido ampliamente en todo el término municipal de Lorca, uno de las de mayor extensión de España, variando desde el grado VII, alcanzado también en algunos de los caseríos o urbanizaciones próximas a Lorca, hasta el grado IV en lugares alejados hasta los $23 \mathrm{~km}$ como la localidad de Morata (figura 6). 


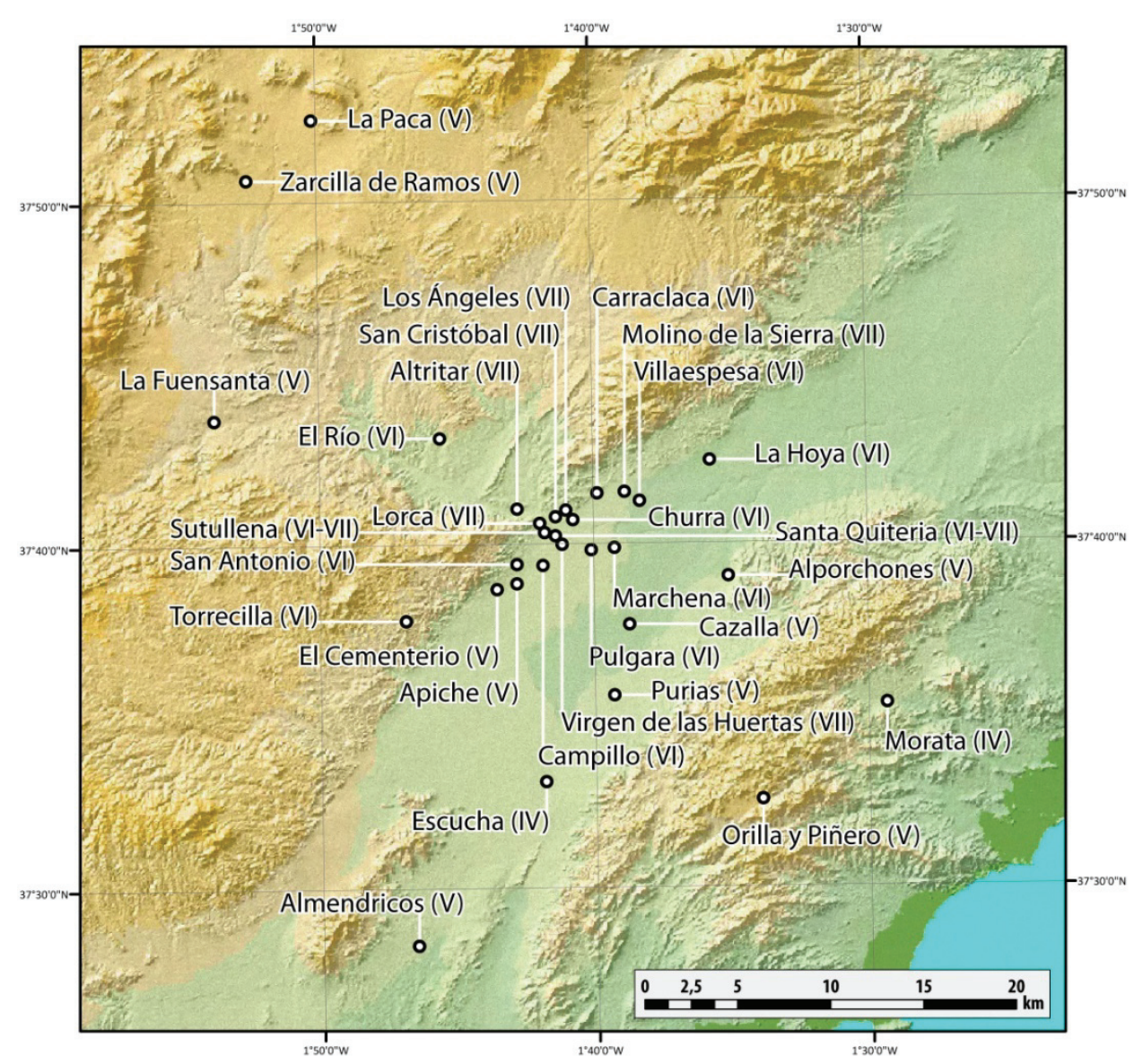

Fig. 6. Intensidades sentidas en localidades del término municipal de Lorca.

Dentro de la región de Murcia, el terremoto no superó el grado V fuera del entorno de Lorca y este valor ha sido asignado a las localidades próximas situadas al noroeste como Alhama de Murcia, Aledo, Mula o Cehegín. Asimismo fue levemente sentido (grado III) en municipios alejados como Jumilla (91 km del epicentro) o Yecla $(112 \mathrm{~km})$ y muy poco perceptible (grado II) en Abanilla $(79 \mathrm{~km})$ o San Pedro del Pinatar $(81 \mathrm{~km})$. Fuera de la región de Murcia el terremoto fue ampliamente sentido en el cuadrante SE peninsular, alcanzando incluso el grado II en Madrid, situada a unos $345 \mathrm{~km}$, o Sevilla a $375 \mathrm{~km}$. La información macrosísmica completa del terremoto y de las réplicas se puede consultar en la página web del IGN.

Como efectos sismogeológicos observados cabe destacar la ocurrencia de desprendimientos de laderas, caídas de rocas de diferentes tamaños o el aumento de 
caudal de algunos manantiales. Estos efectos en la naturaleza son coherentes con la relación que establece la escala EMS98 con la intensidad macrosísmica observada en el epicentro (Grunthal, 1998).

\subsection{Características de la serie sísmica}

En este apartado intentaremos caracterizar los aspectos espaciales y temporales de la serie sísmica. Como se había indicado, se inicia con el sismo premonitorio de magnitud 4,5 ocurrido a las 15:05:13 (GMT) del 11 de mayo de 2011. Durante los siguientes 45 minutos se sucede una serie de 6 sismos premonitorios entre las magnitudes de 1,5 y 2,6. Por el tipo de actividad, la distribución temporal de los premonitorios es del tipo D según la clasificación de Mogi (1985) ya que el terremoto de 5,1 Mw sucede una hora después de una caída de la actividad de la secuencia de los premonitorios. En relación con los eventos posteriores al terremoto principal, si observamos la distribución de epicentros alrededor de la falla de Alhama aparece una dispersión que no permite diferenciar cuales se pueden considerar pertenecientes a la misma serie de réplicas. De igual forma, la sucesión en el tiempo de terremotos en el área, no nos define de forma precisa la duración de la secuencia de réplicas. Para establecer su distribución temporal y espacial de forma objetiva se ha procedido a aplicar una técnica de análisis desarrollada por Alvarez-Gómez et al. (2005). Considerando todos los eventos ocurridos hasta el mes de mayo de 2012 en una zona rectangular de alrededor de Lorca de tamaño 15'x15', este análisis nos ha permitido delimitar la serie temporalmente a unos tres meses aproximadamente, en concreto hasta el 19 de julio de 2011 (figura 7).

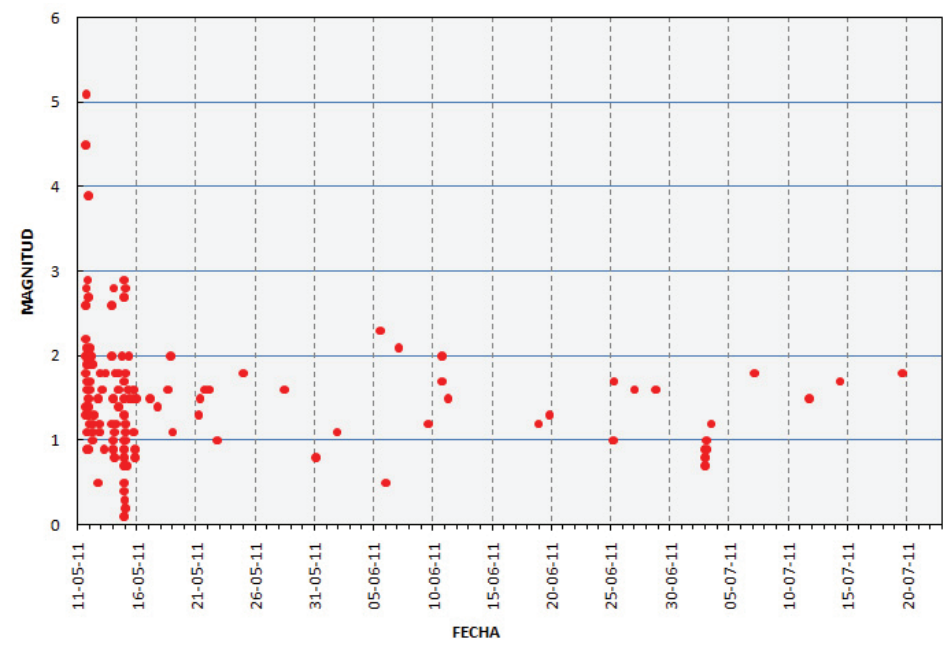

Fig. 7. Evolución temporal de la magnitud de la serie sísmica. 
En total se ha determinado que la serie de réplicas se compone de 141 sismos. Teniendo en cuenta la mayor réplica $(3,9 \mathrm{Mw})$, el parámetro $\mathrm{D}_{1}$, que define la actividad de las replicas y viene dado por $D_{1}=M_{m}-M_{1}$, siendo $M_{m}$ la magnitud del sismo principal y $M_{1}$ la magnitud de esta réplica, se obtiene que $D_{1}=1,2$, es decir se cumpliría la llamada ley de Bath (Utsu, 2002).

Para determinar la relación magnitud-frecuencia, hemos considerado dos conjuntos de terremotos diferentes. Por un lado, la serie de replicas con objeto de poder comparar el parámetro b con el obtenido para la serie de Lorca de 1977 por Mezcua et al. (1984), y por otro, la serie completa incluyendo premonitorios. Los ajustes para calcular la relación magnitud-frecuencia se han estimado siguiendo el método de máxima verosimilitud (Woessner \& Wiemer, 2005), que a partir de un valor mínimo de magnitud 1,6, se ha obtenido para el caso de la serie de réplicas el valor $b=1,02 \pm 0,02$ y $a=3,36$ (figura 8-a). Este valor de $b$ es superior al de 0,67 para la serie de réplicas de Lorca de 1977, (Mezcua et al, 1984) que se determinó mediante un ajuste por mínimos cuadrados, lo que se puede interpretar como una mayor fracturación respecto a la considerada anteriormente. Para la serie completa los valores obtenidos son $b=0,82 \pm 0,19$ y $a=3,09$ (figura 8-b). El valor del parámetro $b$ que hemos calculado se ajusta más para la serie de réplicas que para la serie completa al valor 1,0 dado por el IGN (1992) para la región epicentral.

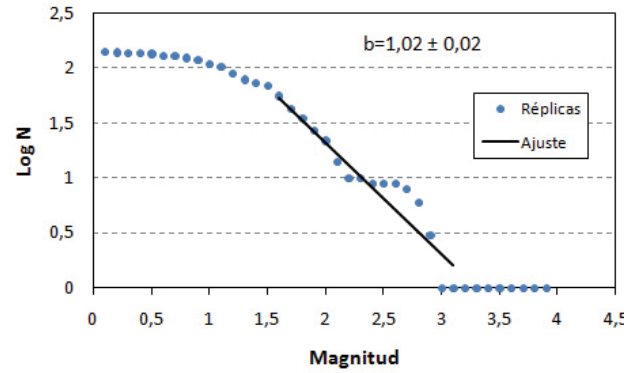

(a)

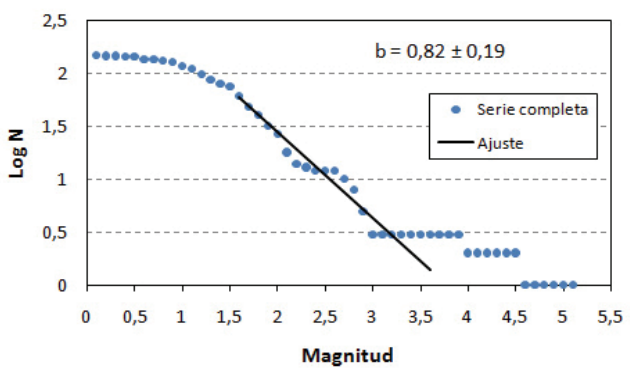

(b)

Fig. 8. Relación magnitud-frecuencia, recta de ajuste y parámetro b para: (a) la serie de réplicas (b) la serie completa.

En relación con la distribución temporal de las réplicas se ha ajustado la serie a la expresión de Omori modificada por Utsu (1962) $n(t)=K(t+c)^{-p}$, donde $n(t)$ es el número de réplicas por día y $\mathrm{K}$, c y $\mathrm{p}$ son constantes. El parámetro $\mathrm{p}$ es independiente del umbral mínimo de magnitud (en este caso $\mathrm{m}=1,6$ ) y expresa la rapidez de la atenuación en frecuencia. El parámetro c, dado en días, está relacionado con un déficit relativo de réplicas ocurridas inmediatamente después del 
terremoto en comparación con una ley de potencia uniforme. Los valores obtenidos son $\mathrm{p}=1,41 \pm 0,16, \mathrm{c}=0,766 \pm 0,355$ y $\mathrm{K}=59,6 \pm 25,6$.

La distribución espacial de la serie, tanto de los premonitorios como de las réplicas, está representada en el mapa de la figura 9, en la que no se han incluido los epicentros con grandes elipses de error. Respecto a la profundidad, existe una dispersión entre 1 y $7 \mathrm{~km}$, estando algunas sin asignar debido también al error obtenido.

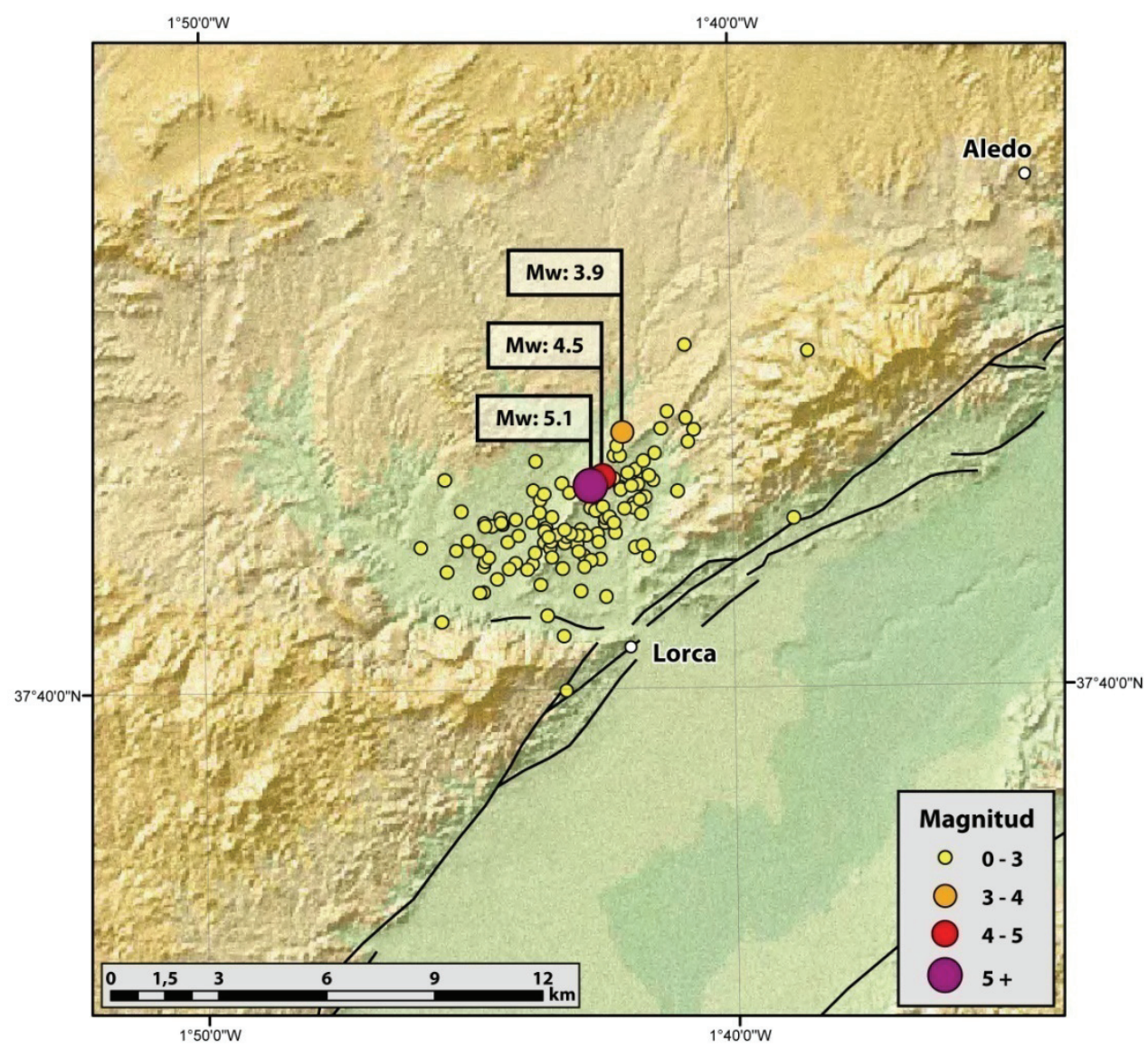

Fig. 9. Localización de los epicentros de la serie sísmica y mecanismo focal de los tres terremotos mayores.

Respecto a la información macrosísmica de la serie, se sintieron 20 réplicas, pero solo la de magnitud 3,9 alcanzó el grado IV (EMS-98), estando el resto por debajo y muchas de ellas sentidas muy levemente con el grado II. 


\section{Sismicidad de la región}

La región donde ha ocurrido el terremoto de Lorca tiene una actividad sísmica moderada dentro del contexto de la península Ibérica, donde se han producido terremotos de intensidad VIII (EMS-98) en épocas históricas y de magnitud máxima registrada de 5,0 en el periodo instrumental, solo superada por el actual sismo de Lorca de 2011. Aunque no debemos restringir el área de estudio de la sismicidad a consideraciones administrativas, hemos tomado como zona la comprendida por la región de Murcia, puesto que las fuentes documentales están referidas a dicha región. Consideramos la sismicidad a partir de intensidad VI en el periodo histórico (tabla 3 ) y de intensidad superior a $\mathrm{V}$ o magnitud mayor de 4,0 para el periodo instrumental (tabla 4), estando reflejada en el mapa de la figura 10, donde se puede apreciar la sucesión de terremotos históricos e instrumentales que han ocurrido a lo largo de la falla de Alhama de Murcia, que hemos señalado es la responsable del reciente terremoto de Lorca. Asimismo, aparecen otros núcleos de actividad notable en la vega del río Segura y puede distinguirse al norte de la citada falla una actividad más reciente $(1999,2002,2005)$, con las magnitudes instrumentales más elevadas.

\subsection{Periodo histórico}

Los terremotos del periodo histórico de intensidad superior a VI reflejados en la tabla 3 se describen brevemente, señalando sus características principales y en las que un análisis más pormenorizado permite algunos cambios de parámetros para los terremotos de 1855 y 1908 .

Tabla 3. Listado de terremotos históricos en la región de Murcia con intensidad superior a VI (Martínez Solares \& Mezcua, 2002).

\begin{tabular}{|l|l|l|l|l|l|}
\hline \multicolumn{1}{|c|}{ Fecha } & $\begin{array}{c}\text { Hora } \\
\text { (GMT) }\end{array}$ & Latitud & Longitud & Imax & Localización \\
\hline $30 / 01 / 1579$ & & 37,6833 & $-1,7000$ & VII & Lorca \\
\hline $28 / 08 / 1674$ & $21: 30: 00$ & 37,6833 & $-1,7000$ & VIII & Lorca \\
\hline $20 / 12 / 1818$ & $09: 45: 00$ & 37,7500 & $-1,6167$ & VI-VII & NE Lorca \\
\hline $11 / 11 / 1855^{*}$ & $04: 00: 00$ & 37,8647 & $-1,3147$ & VI-VII & SE Librilla \\
\hline $16 / 01 / 1883$ & $03: 40: 00$ & 38,0500 & $-1,2500$ & VI-VII & Ceutí \\
\hline $26 / 09 / 1908^{*}$ & $09: 50: 10$ & 38,0685 & $-1,3950$ & VI-VII & NE Mula \\
\hline
\end{tabular}

* Modificado 


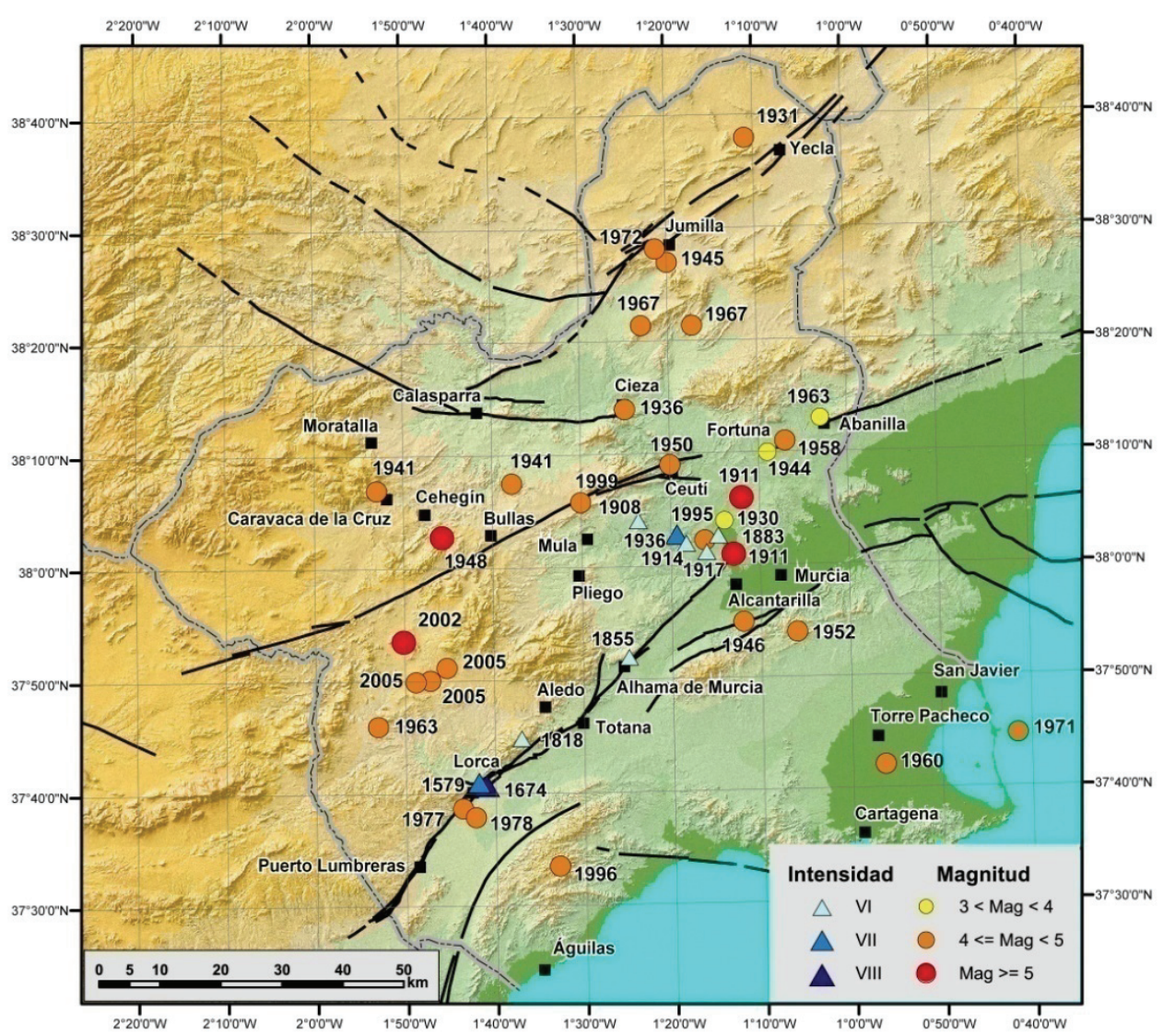

Fig. 10. Sismicidad de la región de Murcia para los terremotos históricos con $\mathrm{I}>\mathrm{V}$ e instrumentales con $\mathrm{I}>\mathrm{V}$ o magnitud $\geq 4.0$.

Terremoto de Lorca de 1579

Este terremoto, no referenciado en el catálogo de Galbis Rodríguez (1932), lo cita Martínez Guevara (1984) de un documento extraído del Archivo Municipal de Lorca en el que se notifica que se produjo en Lorca en 1579 una serie sísmica sin poder especificar su duración, con un terremoto más intenso el 30 de enero durante el cual muchos vecinos abandonaron la ciudad por haber quedado las "casas $y$ edificios muy a peligro y arruinadas y muchas dellas caydas..." .

Terremoto de Lorca de 1674

Durante el año 1674 se produce de nuevo una serie sísmica próxima a Lorca de casi dos meses de duración, que se inicia el 10 de agosto con un terremoto pre- 
monitorio de intensidad V-VI. El terremoto principal sucede el día 28 de agosto a las $21 \mathrm{~h} 30 \mathrm{~m}$, estimándose una intensidad de VIII dado que los daños en algunas edificaciones llegaron a ser muy graves como refleja la tabla 4 , que cuantifica los daños en diversas parroquias de Lorca.

Al día siguiente se repitió la sacudida, así como los días 9 de septiembre y 5 de octubre. El número de víctimas mortales se estima que fue superior a 30. (Martínez Guevara \& Fernández-Navarro Soto, 1986; Vidal, 1986; Martínez Solares \& Mezcua, 2002).

Tabla 4. Daños ocasionados por el terremoto de 1674 distribuidos por parroquias de Lorca (Vidal, 1986).

\begin{tabular}{|l|c|c|c|c|}
\hline \multicolumn{1}{|c|}{ PARROQUIA } & Santiago & San Mateo & $\begin{array}{l}\text { Santa María, } \\
\text { San Patricio, } \\
\text { San Juan y } \\
\text { San Pedro }\end{array}$ & San Cristóbal \\
\hline Edificios reconocidos & 282 & 594 & 415 & 348 \\
\hline Edificios asolados & 56 & 73 & 8 & 83 \\
\hline $\begin{array}{l}\text { Edificios con daños } \\
\text { superiores a mil reales }\end{array}$ & 217 & 367 & 117 & - \\
\hline $\begin{array}{l}\text { Edificios can daños } \\
\text { inferiores a mil reales }\end{array}$ & 9 & 132 & 6 & 264 \\
\hline Sin daños & 0 & 22 & - & 1 \\
\hline
\end{tabular}

Terremoto de Lorca de 1818

El día 19 de diciembre alrededor de las $16 \mathrm{~h}$ se produce un sismo, también premonitorio, de intensidad IV con epicentro al NE de Lorca. Al día siguiente, a las $9 \mathrm{~h} 45 \mathrm{~m}$ ocurre el terremoto principal, sentido en Lorca, Totana y Murcia capital y cuyos daños no fueron demasiado graves, pudiendo asignarse una intensidad de VI-VII (Martínez Guevara \& Fernández-Navarro Soto, 1986; Martínez Solares \& Mezcua, 2002). Según anota Rodríguez de la Torre (1993) "dos personas quedaron muy mal heridas entre los escombros" y observándose asimismo efectos sismogeológicos como el "hundimiento de tierras" entre Lorca y Totana.

\section{Terremoto de Librilla de 1855}

Este terremoto catalogado por Martínez Solares \& Mezcua (2002) en Alhama de Murcia ha sido nuevamente evaluado en este trabajo trasladando su localización epicentral al SE de Librilla donde los daños han sido mayores (Martínez Guevara, 1984). Este terremoto produjó un muerto causado por el hundimiento de una cueva en Los Zancarrones, lugar próximo a Librilla. 


\section{Terremoto de Ceutí de 1883}

El terremoto de 16 de enero es el de mayor intensidad dentro de una serie sísmica localizada en la vega del río Segura próxima a Ceutí y Archena que comenzó en octubre del año anterior (1882) y que finalizó en febrero de 1883. En Ceutí se registraron daños en algunas viviendas (Fernández Navarro-Soto, 1985).

\section{Terremoto de Mula de 1908}

En el catálogo de Mezcua \& Martínez Solares (1983) figuran dos terremotos los días 26 y 29 de septiembre de 1908. La fuente procede de Galbis Rodríguez (1932) que anota los dos sismos, el segundo sin especificar hora y citando fuentes que parecen ser susceptibles de confusión en la fecha. Como solo el del día 26 fue registrado por el Observatorio de Cartuja y considerando la información macrosísmica, estimamos que se trata de un único terremoto acaecido el día 26 a las 9:50 (GMT) sentido en Mula, Abarán y Ojós y con epicentro al NE de Mula. Con los datos macrosísmicos aportados por Galbis Rodríguez (1932) y Comas Solá (1909) podemos asignar una intensidad VI-VII (EMS98).

\subsection{Periodo instrumental}

Consideramos este periodo como el que tiene registros instrumentales al menos en tres estaciones u observatorios, independientemente de que el cálculo hipocentral haya sido realizado a partir de las lecturas de los registros o mediante la información macrosísmica o de que disponga de magnitud instrumental. La tabla 5 refleja los terremotos de este periodo para la región de Murcia con intensidad superior a V o magnitud superior a 4,0 obtenidos del catálogo de Mezcua \& Martínez Solares (1983) y de la base de datos del IGN. No obstante, algunos de los terremotos de la tabla han sido revisados y en consecuencia se ha modificado alguno de sus parámetros.

La serie de 1911, que estuvo activa entre marzo y mayo, ha sido estudiada por Buforn et al. (2005) que han rebajado la intensidad del terremoto de 21 de marzo al grado VII-VIII. Asimismo, las magnitudes fueron recalculadas con valores de Ms 5,7 y 5,3, para los sismos de marzo y abril, respectivamente. El terremoto de 1917, con la información aportada por Fontsere (1917), permite también rebajar un grado el valor de la intensidad respecto al grado VII del catálogo original. El 3 de septiembre de 1930 figura el terremoto con un grado VII dado por Poyato (1930) y que se ha mantenido en los siguientes catálogos. Sin embargo, haciendo una lectura más crítica del texto, el daño que se produce llegaría a alcanzar como mucho al grado 2 (EMS-98) y en edificios de vulnerabilidad alta y en mal estado de conservación, por tanto estimamos que la intensidad debe ser de grado VI (EMS98).

En el año 1941, el 24 de noviembre se produce un terremoto con magnitud 4,1 que es registrado en cinco observatorios españoles, lo que ha permitido realizar un cálculo hipocentral mediante el programa HYPO (Lee \& Lahr, 1975) y el modelo de corteza de Mezcua \& Martínez Solares (1983). Las coordenadas epicentrales 
obtenidas usando 9 lecturas son las reflejadas en la tabla 5, con una profundidad del foco de $10 \mathrm{~km}$ y un RMS de 1,34. También la localización se ha desplazado al este de Caravaca y la intensidad máxima ha sido modificada al grado V-VI (Rey Pastor, 1951).

Un terremoto más significativo por su magnitud $(5,0)$ que el anterior, el del 23 de junio de 1948, ha sido también relocalizado instrumentalmente a partir de 12 lecturas de ocho observatorios incluido el de Lisboa, obteniéndose una nuevas coordenadas epicentrales (ver tabla 5). La profundidad hipocentral se ha situado a $5 \mathrm{~km}$, el RMS de las lecturas es 2,09, valor que consideramos aceptable para ese año. Este sismo fue sentido ampliamente en las provincias de Murcia y Albacete, alcanzando según Rey Pastor (1949) el grado VIII, escala Forell-Mercalli-Sieberg, aunque rebajamos esta intensidad al grado VII (EMS98), puesto que si bien en Cehegín los daños ocasionados en un pequeño barrio hizo que se tuviera que derribar por completo, sus casas eran viejas y estaban mal construidas. Hubo efectos sismogeológicos como la caída de piedras, rotura de rocas o aumento de caudal en los manantiales. La intensidad sísmica del terremoto del 22 de diciembre de 1958 ha sido también levemente modificada a la baja asignándole el grado V-VI (Due Rojo, 1960).

Tabla 5. Terremotos con registro instrumental en la región de Murcia con intensidad $>\mathrm{V}$ ó magnitud $\geq 4.0$. (Mezcua \& Martínez Solares, 1983; Base de Datos IGN)

\begin{tabular}{|l|l|l|l|l|l|l|}
\hline FECHA & HORA & LAT & LONG & $\begin{array}{l}\text { MAG } \\
(\text { Tipo })^{* *}\end{array}$ & INT & LOCALIZACION \\
\hline $21 / 03 / 1911$ & $14: 15: 35$ & 38,0167 & $-1,2167$ & $5,7-(6)$ & VII-VIII* & Torres de Cotillas \\
\hline $03 / 04 / 1911$ & $11: 11: 11$ & 38,1000 & $-1,2000$ & $5,3-(6)$ & VIII & Lorquí \\
\hline $17 / 03 / 1914$ & $19: 18: 56$ & 38,0333 & $-1,3333$ & & VI & Campos del Rio \\
\hline $28 / 01 / 1917$ & $22: 32: 31$ & 38,0333 & $-1,2667$ & & VI* & Torres de Cotillas \\
\hline $03 / 09 / 1930$ & $09: 59: 58$ & 38.0667 & $-1,2333$ & $3,7-(1)$ & VI* & Lorquí \\
\hline $26 / 01 / 1931$ & $03: 16: 15$ & 38.6333 & $-1,1833$ & $4,0-(1)$ & VI & Yecla \\
\hline $30 / 01 / 1936$ & $09: 35: 18$ & 38,0667 & $-1,2667$ & & VII & Ceutí \\
\hline $13 / 06 / 1936$ & $00: 36: 59$ & 38,2333 & $-1,4167$ & $4,5-(1)$ & IV & Cieza \\
\hline $26 / 10 / 1941$ & $05: 19: 27$ & 38,1167 & $-1,8833$ & $3,9-(1)$ & VI & Caravaca \\
\hline $24 / 11 / 1941$ & $00: 53: 59^{*}$ & $38,1250^{*}$ & $-1,6300^{*}$ & $4,1-(1)$ & V-VI* & E Caravaca* \\
\hline $23 / 02 / 1944$ & $22: 34: 10$ & 38,1667 & $-1,1500$ & $3,8-(1)$ & VII & Fortuna \\
\hline $14 / 05 / 1945$ & $06: 33: 30$ & 38,4500 & $-1,3333$ & $4,3-(1)$ & VI & Jumilla \\
\hline $14 / 05 / 1946$ & $12: 21: 20$ & 37,9167 & $-1,2000$ & $4,2-(1)$ & VI & Sangonera \\
\hline $23 / 06 / 1948$ & $03: 43: 58^{*}$ & $38,0467^{*}$ & $-1,7617^{*}$ & $5,0-(1)$ & VII* & Cehegin \\
\hline $02 / 05 / 1950$ & $07: 37: 46$ & 38,1500 & $-1,3333$ & $4,0-(1)$ & VI & Archena \\
\hline
\end{tabular}




\begin{tabular}{|l|l|l|l|l|l|l|}
\hline $20 / 05 / 1952$ & $13: 18: 05$ & 37,9000 & $-1,1000$ & $4,4-(1)$ & V & Palmar \\
\hline $22 / 12 / 1958$ & $02: 48: 16$ & 38,1833 & $-1,1167$ & $4,0-(1)$ & V-VI* & Fortuna \\
\hline $01 / 11 / 1960$ & $10: 56: 21$ & 37,7017 & $-0,9400$ & $4,0-(1)$ & V & SE Torre-Pacheco \\
\hline $19 / 01 / 1963$ & $20: 50: 29$ & 38,2167 & $-1,0500$ & $3,3-(2)$ & VI & Abanilla \\
\hline $30 / 05 / 1963$ & $01: 54: 49$ & 37,7667 & $-1,8850$ & $4,0-(2)$ & V & NW Lorca \\
\hline $29 / 07 / 1967$ & $21: 54: 29$ & 38,3567 & $-1,3833$ & $4,2-(2)$ & III & NE Cieza \\
\hline $03 / 08 / 1967$ & $00: 34: 13$ & 38,3567 & $-1,2883$ & $3,9-(2)$ & VI & SE Jumilla \\
\hline $12 / 01 / 1971$ & $05: 07: 13$ & 37,7450 & $-0,6933$ & $4,2-(2)$ & V & SE S. P. del Pinatar \\
\hline $14 / 04 / 1972$ & $03: 22: 17$ & 38,4700 & $-1,3550$ & $4,2-(2)$ & & Jumilla \\
\hline $06 / 06 / 1977$ & $10: 49: 12$ & 37,6450 & $-1,7283$ & $4,2-(2)$ & VI & SW Lorca \\
\hline $24 / 03 / 1978$ & $13: 01: 24$ & 37,6317 & $-1,7050$ & $4,3-(2)$ & & S Lorca \\
\hline $26 / 11 / 1995$ & $05: 39: 40$ & 38,0383 & $-1,2700$ & $4,1-(2)$ & VI-VII & SW Alguazas \\
\hline $02 / 09 / 1996$ & $19: 07: 01$ & 37,5583 & $-1,5500$ & $4,5-(2)$ & V & N Águilas \\
\hline $02 / 02 / 1999$ & $13: 45: 17$ & 38,0963 & $-1,5014$ & $4,7-(2)$ & VI & N Mula \\
\hline $06 / 08 / 2002$ & $06: 16: 19$ & 37,8925 & $-1,8353$ & $5,0-(5)$ & V & SW Bullas \\
\hline $29 / 01 / 2005$ & $07: 41: 32$ & 37,8535 & $-1,7555$ & $4,8-(5)$ & VII & NW Aledo \\
\hline $03 / 02 / 2005$ & $11: 40: 33$ & 37,8349 & $-1,7864$ & $4,3-(5)$ & IV-V & NW Lorca \\
\hline $04 / 02 / 2005$ & $01: 09: 41$ & 37,8325 & $-1,8135$ & $4,0-(5)$ & III-IV & N Lorca \\
\hline
\end{tabular}

* Modificaciones respecto al catalogo

**Tipo de magnitud: (1) Magnitud por duración (Mezcua \& Martínez Solares, 1983). (2) Magnitud a partir de la fase Lg (Mezcua \& Martínez Solares, 1983). (5) Magnitud momento (Rueda \& Mezcua, 2005). (6) (Buforn et al. 2005).

Por lo que respecta al periodo instrumental más reciente, se ha registrado un aumento de la actividad sísmica de la región, que ha coincidido además con la instalación de nuevas estaciones de banda ancha que han ampliado la capacidad de la red sísmica y la calidad de los datos registrados. Este nivel de actividad se inicia con el terremoto ocurrido el 2 de febrero de 1999 al norte de Mula (Murcia) de magnitud $\mathrm{m}_{\mathrm{bLg}} 4,7$ que tuvo un premonitorio, $\mathrm{m}_{\mathrm{bLg}} 3,5$, ocurrido 23 minutos antes. La serie tuvo una duración de dos meses y la intensidad máxima sentida fue de VI, con el mayor daño reportado en las localidades de Mula, Las Torres de Cotillas y Campos del Rio. La aceleración pico registrada en la estación más próxima (Lorquí, $21 \mathrm{~km}$ ) fue de $0,013 \mathrm{~g}$ (componente E-W). Otra serie sísmica se inicia en Bullas, pueblo próximo a Mula, el día 6 de agosto de 2002 con un terremoto de magnitud 5,0 (Mw). Seguramente, la gran distancia del epicentro a la población hizo que la máxima intensidad sentida fuese solo de grado $\mathrm{V}$, siendo las localidades de Bullas y Cehegín las que más lo percibieron. La máxima aceleración se registró en el equipo del IGN ubicado en Mula (a $32 \mathrm{~km}$ del epicentro) con un valor de 0,020g (componente E-W). Este terremoto ha sido estudiado por Buforn et al.(2005). Por último, el terremoto de 29 de enero de 2005 en La Paca, 
término municipal de Aledo, de magnitud 4,8 $(\mathrm{Mw})$ es el inicio de una larga serie que se puede considerar finalizada en el mes de marzo. Las poblaciones más afectadas fueron La Paca y Zarcilla de Ramos, donde el sismo principal alcanzó el grado VII (Benito et al. 2007). La máxima aceleración se registró en Mula a una distancia de $27 \mathrm{~km}$ del epicentro, con valor de $0,024 \mathrm{~g}$ (componente $\mathrm{N}-\mathrm{S}$ ).

Como resumen de la sismicidad de la región hemos representado en el mapa de la figura 11, la distribución de los epicentros de magnitud $\mathrm{Mw}$ superior a 3,0 en los que se han transformado todos los valores de intensidad y magnitud en sus diferentes tipos a magnitudes $\mathrm{Mw}$ (Cabañas et al. 2012), recogiendo también las áreas próximas de Alicante, Albacete y Almería. El mayor nivel de magnitud entre 5,0 y 6,0 se localiza a lo largo de la falla de Alhama de Murcia y en la vega media del río Segura, si bien un nivel de magnitud superior está localizado fuera de la región en las áreas de Torrevieja y Vera.

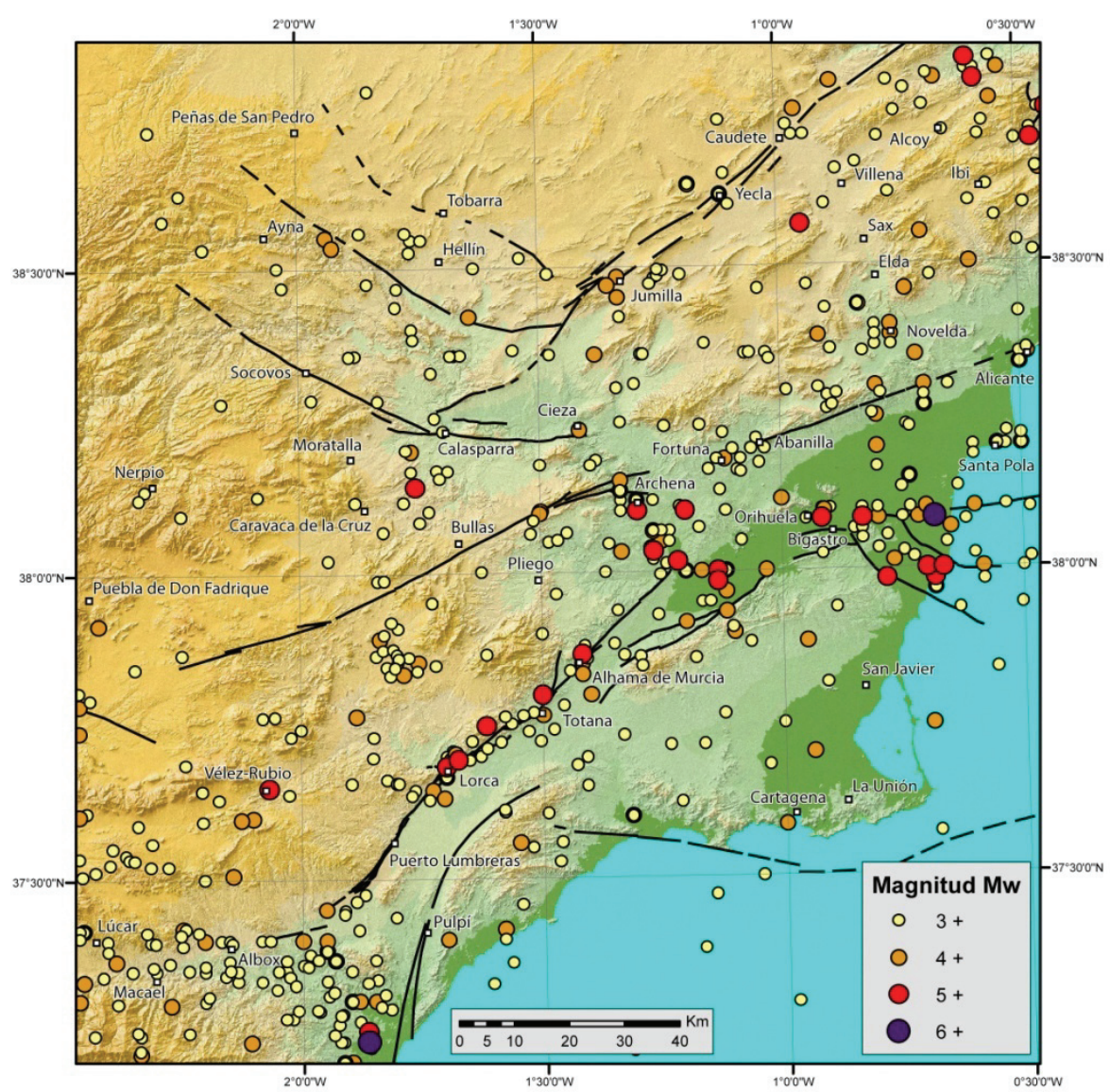

Fig. 11. Sismicidad de la región de Murcia en valores de magnitud momento desde 3.0. 


\section{Conclusiones}

El registro de la serie sísmica en equipos de banda ancha y acelerométricos con una buena distribución acimutal nos ha permitido determinar de forma precisa las localizaciones hipocentrales de la serie, en la que hemos eliminado las estaciones situadas a distancias superiores a $\operatorname{los} 4^{\circ}$ con objeto de minimizar los errores que se inducen al manejar un solo modelo de corteza. Asimismo, una nueva determinación del tensor momento sísmico ha sido realizada utilizando 21 estaciones, mejorando notablemente la anterior dada por el IGN que había sido calculada automáticamente solamente con tres estaciones. Los mecanismos focales obtenidos son consistentes con las características de la falla de Alhama de Murcia, con un movimiento de falla inversa con ligera componente de desgarre.

La densificación de equipos acelerométricos en la zona ha permitido aumentar notablemente el número de registros de aceleración para un mismo terremoto y obtener el máximo valor de aceleración registrado hasta ahora en España, un valor de $0,36 \mathrm{~g}$ muy superior al que hasta ahora se había obtenido de $0,024 \mathrm{~g}$ para la zona con el terremoto de Aledo (4,8 Mw) en 2005. Este alto valor de aceleración a una distancia hipocentral próxima a la fuente de $6 \mathrm{~km}$, obligará a modificar los modelos de atenuación del movimiento fuerte para la zona. Por otro lado, un futuro incremento de estos equipos en la zona, unido al aumento de los puntos de información macrosísmica evaluados de forma automática vía internet, permitirá realizar mapas de sacudida en tiempo casi real de gran utilidad para los sistemas de alerta y de protección civil.

Un nuevo análisis de la sismicidad ha llevado a modificar los parámetros de algunos terremotos históricos, lo que pone de manifiesto la necesidad de continuar investigando en todo tipo de archivos. Por lo que respecta al periodo instrumental, se ha realizado un intento de determinar mediante programas de cálculo el hipocentro de los terremotos de la tabla 5 anteriores a 1962, considerada una fecha a partir de la cual existe una mayor fiabilidad en las lecturas debido a la instalación de las tres estaciones estándar WWSSN de la península (TOL, MAL, PTO). Se ha partido de los datos de lectura de los boletines sísmicos que confeccionaban los propios observatorios, al considerarlos más fiables que los dados en los boletines generales que se publicaban anualmente. También, debido a los errores en las lecturas de las diferentes fases inherentes a la escasa resolución temporal y a la posible mala interpretación de llegadas secundarias anotadas en los boletines, se ha decidido usar solamente las primeras llegadas de las diferentes fases de la onda $\mathrm{P}$ y su correspondiente fase en la $\mathrm{S}$, lo que ha motivado el uso del programa HYPO, que solo usa dos fases por estación. En cualquier caso, el resultado ha sido escaso y, como se apuntó en el apartado anterior, solamente dos terremotos han podido ser calculados de forma fiable. Esto pone de manifiesto la necesidad de acudir a los sismogramas originales, pues algunas de las dificultades planteadas pueden ser resueltas.

En general la ocurrencia de eventos premonitorios, como ha sucedido en Lorca en 2011, es mucho menos frecuente comparada con la de las réplicas, no obstante en la región de Murcia, tanto en el periodo histórico como instrumental, muchos 
de los grandes terremotos (> VI) presentan premonitorios, siendo el intervalo de tiempo muy variable, desde una quincena de días aproximadamente para los terremotos de 1674 (VIII-EMS) y 1911 (5,3 Ms), hasta unas pocas horas (1818, VI-VII ; 2011, 5,1 Mw) e incluso 23 minutos, como sucedió con el de 1999 de Mula (4,7 mbLg). En todos estos casos el tipo de actividad corresponde al tipo D definido por Mogi (1985). Por otro lado, los premonitorios están asociados con heterogeneidades en el material de la fuente, resultando que las secuencias de replicas tienen una mayor duración (Udías, 1999), lo que sucede con los terremotos de Lorca de 1674 y 2011. En el caso de contrario, sin eventos premonitorios como los de Bullas de 2002 (5,0 Mw) y de Aledo de 2005 (4,8 Mw) las series de réplicas llegaron a superar los dos meses, si bien ambos sismos estuvieron localizados en la misma falla y próximos entre sí e incluso con un mecanismo de disparo entre ellos como apuntan Benito et al. (2007).

\section{Agradecimientos}

Agradecemos a las redes sísmicas del Instituto Andaluz de Geofísica, del Real Observatorio de la Armada - Universidad Complutense de Madrid y de la Universidad de Alicante su colaboración al poner a nuestra disposición los datos de sus estaciones sísmicas.

\section{Referencias bibliográficas}

ÁLVAREZ-GÓMEZ, J.A., GARCIA-MAYORDOMO, J., MARTÍNEZ DÍAZ, J.J. \& CAPOTE, R. (2005). SeriesBuster: a Matlab program to extract spatiotemporal series from an earthquake database. Computers \& Geosciences 31, $521-525$.

BENITO, B., CAPOTE, B., MURPHY, P., GASPAR-ESCRIBANO, J.M., MARTÍNEZ-DÍAZ, J.M., TSIGE, M., STICH, D., GARCÍA MAYORDOMO, J., GARCÍA-RODRÍGUEZ, M.J., JIMÉNEZ PEÑA, M.E., INSUAARÉVALO, J.M., ÁLVAREZ-GÓMEZ, J.A. \& CANORA, C. (2007). An Overview of the Damaging and Low Magnitude Mw 4.8 La Paca Earthquake on 29 January 2005: Context, Seismotectonics, and Seismic Risk Implications for Southeast Spain. Bull. Seismol. Soc. Am. 97, 671-690.

BUFORN, E., BENITO, B., SANZ DE GALDEANO, C., DEL FRESNO, C., MUÑOZ, D. \& RODRÍGUEZ, I. (2005). Study of the damaging earthquakes of 1911, 1999, and 2002 in the Murcia, Southeastern Spain, region: seismotectonic and seismic-risk implications. Bull. Seism. Soc. Am. 95, 549-567.

CABAÑAS, L., RIVAS, A., MARTÍNEZ-SOLARES, J.M., GASPARESCRIBANO, J., BENITO, B., ANTÓN, R. \& RUIZ-BARAJAS, S. (2012). Preparación y homogeneización de un catálogo sísmico para la evaluación de la peligrosidad sísmica en España. $7^{a}$ Asamblea Hispano-Portuguesa de Geodesia y Geofísica. San Sebastián, 25-29 junio de 2012. 
COMAS SOLÁ, J. (1909). Observaciones sísmicas efectuadas durante el año 1908. Mem. de la Real Academia de Ciencias y Artes de Barcelona. Vol. VII, $13,532-543$.

DUE ROJO, A. (1960). Movimientos sísmicos en España durante el año 1958. Bol. R. Soc. Hist. Nat. T. LVIII, 97-104.

FERNÁNDEZ NAVARRO-SOTO, S. (1985). Más información sobre terremotos acontecidos durante el siglo XIX en la región de Murcia. Estudio de sismicidad histórica. Informe interno, Instituto Geográfico Nacional, $32 \mathrm{pp}$.

FONTSERÉ, E. (1917). Nota sobre el temblor de tierra ocurrido en Cotillas (Murcia) el 28 de enero de 1917. Mem. de la Real Academia de Ciencias y Artes de Barcelona. Vol. XIII, 16, 233-237.

GALBIS RODRÍGUEZ, J. (1932). Catálogo sísmico de la zona comprendida entre los meridianos $5^{\circ} \mathrm{E}$ y $20^{\circ} \mathrm{W}$ y paralelos $45^{\circ}$ y $25^{\circ} \mathrm{N}$. Instituto Geográfico y Catastral, Tomo I, $807 \mathrm{pp}$.

GRUNTHAL, G. (ed.) (1998). European Macroseismic Scale 1998. Cahiers du Centre Europeen de Geodynamique et de Seismologie. Vol. 15, 99 pp.

HERRMANN, R.B. \& AMMON, C. (2002). Computer programs in seismology 3.30: Source inversion. In: www.eas.slu.edu/People/RBHerrmann /CPS330.html.

INSTITUTO GEOGRÁFICO NACIONAL (1992). Análisis sismotectonico de la península Ibérica, Baleares y Canarias. Instituto Geográfico Nacional, Pub. Técnica 26, 43 pp + 1 mapa.

LEE, W.H.K. \& J.C. LAHR (1975). HYPO 71: A computer program for determining hypocenters magnitude and first motion pattern of earthquake. U.S. Geol. SUN. Open-File Rep.

MARTÍNEZ DÍAZ, J.J. (2002). Stress field variety related to fault interaction in a reverse oblique-slip fault: The Alhama de Murcia fault, Betic Cordillera, Spain. Tectonophysics, 356, 291-305.

MARTÍNEZ GUEVARA, J.B. (1984). Temblores de tierra en el núcleo sísmico Lorca-Totana (Murcia). Informe interno, Instituto Geográfico Nacional, $51 \mathrm{pp}$.

MARTÍNEZ GUEVARA, J.B. y FERNÁNDEZ NAVARRO-SOTO, S. (1986). Catálogo sísmico de la región de Murcia. (Sismicidad histórica hasta el siglo $X V I I I)$. Informe interno, Instituto Geográfico Nacional, $71 \mathrm{pp}$.

MARTÍNEZ SOLARES, J.M. \& MEZCUA, J. (2002). Catalogo sísmico de la Península Ibérica (880 a.C. - 1900). Instituto Geográfico Nacional, 253 pp + 1 mapa.

MEZCUA, J., HERRAIZ, M. \& BUFORN, E. (1984). Study of the 6 June 1977 Lorca (Spain) earthquake and its aftershock sequence. Bull. Seism. Soc. Am. 74, 167-179.

MEZCUA, J. \& MARTÍNEZ SOLARES, J.M. (1983). Sismicidad del área Iberomogrebi. Instituto Geográfico Nacional, $301 \mathrm{pp}+1$ mapa.

MOGI, K. (1985). Earthquake prediction. Academic Press, New York. 
NCSE-02. (2002). Norma de Construcción Sismorresistente: Parte general y edificación. BOE, 244, 35898-35967.

POYATO, J. (1930). Nota sobre el sismo del día 3 de septiembre de 1930 (Lorqui). Informe interno, Instituto Geográfico y Catastral, $14 \mathrm{pp}$.

REY PASTOR, A. (1949). La comarca sísmica de Caravaca y el sismo de 23-junio-1948. Instituto Geográfico y Catastral, $35 \mathrm{pp}$.

REY PASTOR, A. (1951). Estudio sismotectónico de la región sureste de España. Instituto Geográfico y Catastral, $52 \mathrm{pp}$.

RODRÍGUEZ DE LA TORRE, F. (1993). Revisión del catálogo sísmico ibérico. Años 1801 a 1850. Informe interno, Instituto Geográfico Nacional.

RUEDA, J. \& MEZCUA, J. (2005). Near-real-time seismic moment-tensor determination in Spain. Seismol. Res. Lett. 76, 455-465.

RUEDA, J., MEZCUA, J. \& GARCÍA BLANCO, R.M. (2011). Directivity effects of the May 11, 2011 Lorca (Spain) Mw=5.1 earthquake. American Geophysical Union, Fall Meeting 2011, abstract \#S52B-2277.

UDÍAS, A. (1999). Principles of Seismology. Cambridge University Press, 475 pp.

UTSU, T. (1962). On nature of three Alaskan aftershock sequences of 1957 and 1958. Bull. Seism. Soc. Am. 52, 279-297.

UTSU, T. (2002). Statistical features of seismicity. in W. H. K. Lee, H. Kanamori, P.C.Jennings, \& C. Kisslinger (eds.) International Handbook of Earthquake \& Engineering Seismology Part A, Academic Press, San Diego, 719-732.

VIDAL, F. (1986). Sismotectónica de la región Béticas- Mar de Alborán. Tesis Doctoral, Universidad de Granada, $450 \mathrm{pp}$.

WOESSNER, J. \& WIEMER, S. (2005). Assessing the Quality of Earthquake Catalogues: Estimating the Magnitude of Completeness and Its Uncertainty. Bull. Seism. Soc. Am. 95, 684-698. 